\title{
Dynamics of a low-threshold optically pumped organic vertical-cavity surface-emitting laser
}

\author{
Mohammad Reza Shayesteh ${ }^{1}$ - Ghafar Darvish ${ }^{1}$
}

Received: 12 September 2015/Accepted: 5 January 2016/Published online: 28 January 2016

(C) The Author(s) 2016. This article is published with open access at Springerlink.com

\begin{abstract}
We propose a low-threshold optically pumped organic vertical-cavity surface-emitting laser (OVCSEL). This device has the capability to apply both electrical and optical excitation. The microcavity structure consists of an organic light emitting diode with field-effect electron transport inserted in a high-quality factor double distributed Bragg reflector. The simulated quality factor of the microcavity is shown to be as high as 16,000 . Also, we investigate threshold behaviour and the dynamics of the optically pumped OVCSEL with sub-picosecond pulses. Results from numerical simulation show that lasing threshold is $12.8 \mathrm{pJ} / 0.64 \mu \mathrm{J} \mathrm{cm}^{-2}$ when pumped by subpicosecond pulses of $\lambda=400 \mathrm{~nm}$ wavelength light.
\end{abstract}

Keywords Organic semiconductor - Organic verticalcavity surface-emitting laser (OVCSEL) - Optical pump · Quality factor · Lasing threshold

\section{Introduction}

Organic semiconductor lasers provide superior features such as flexibility, accessibility of all wavelengths in the visible spectrum, and possibility of low cost and large-area

Ghafar Darvish

darvish_gh@srbiau.ac.ir

Mohammad Reza Shayesteh

m_shayesteh45@yahoo.com

1 Department of Electrical Engineering, Science and Research Branch, Islamic Azad University, Tehran, Iran fabrication. These properties would make organic semiconductor lasers suitable for many applications in bioanalytics, digital printing, and fluorescence spectroscopy [1]. Although the first optically pumped organic laser has been demonstrated as early as 1996 [2], an electrically pumped organic laser has not been realized yet. Low charge carrier mobility, bimolecular annihilations process, induced absorption process, field quenching, and metal contact losses are the important factors that prevent lasing. Moreover, electrode contact absorption losses are most common losses in organic laser diodes. To develop an effective electrically pumped organic laser, one part of the strategy is lowering the optical losses. The various optically pumped organic microcavity geometries such as double distributed Bragg reflectors (DBRs) [3, 4], distributed feedback structures [5, 6], 2D photonic crystals [7, 8], photonic band gap fiber resonators [9], photonic crystal nanobeam cavity [10], and high contrast grating (HCG)based microcavity [11] have been demonstrated. For optically pumped organic laser, microcavity with a highquality factor leads to a lower optical power input. Chakaroun et al. proposed a high-quality factor microcavity organic laser for electrical pumping [12]. They have used high-reflection and low-absorption DBR mirror layers for reaching high-quality factor.

In this paper, we propose a low-threshold OVCSEL which has the feasibility to apply both electrical and optical pumping. The extended microcavity structure consists of an organic light emitting diode (OLED) with field-effect electron transport [13] set in between DBR mirrors. We also model the threshold behaviour and the dynamics of the OVCSEL optically pumped with sub-picosecond pulses. The results of optical pump-out will help us to estimate the injection current density value for electrical pumping. 


\section{Organic VCSEL structure design}

The schematic structure of the proposed OVCSEL is shown in Fig. 1. The OLED with field-effect electron transport has been used as the active region. In our device structure, the metallic top contact is remote from the light-emission zone. As a result, the additional losses due to absorption photon at the metal contact can be decreased. Therefore, the quality factor of the microcavity that is inversely proportional to the cavity loss coefficient can be increased. The exciton density at the laser threshold $(N$ th) is reduced with a high-quality factor. The active region consists of several organic layers, including the host-guest system of tris-(8-hydroxyquinoline) aluminum $\left(\mathrm{Alq}_{3}\right)$ doped with 4-(dicyanomethylene)-2-methyl-6-( $p$-dimethylaminos-

tyryl)-4H-pyran (DCM) with a thickness of $40 \mathrm{~nm}$ as the emission layer (EML), $N, N^{\prime}$-ditridecylperylene-3,4,9,10tetracarboxylic diimide (PTCDI- $\mathrm{C}_{13} \mathrm{H}_{27}$ ) with a thickness of $40 \mathrm{~nm}$ as the electron transport (ETL) and hole blocking (HBL) layer, and poly(triarylamine) (PTAA) with a thickness of $80 \mathrm{~nm}$ as the hole transport layer (HTL). The host-guest system as the emission layer has several advantages: (1) the host molecules efficiently absorb the pump light and non-radiatively transfer it via a Förster process to the guest molecules with efficiency of more than $90 \%$ [14] and (2) the low concentration of the guest molecules reduces bimolecular annihilation and hence lowers the laser threshold.

The chosen anode is an indium tin oxide (ITO) $(12 \mathrm{~nm}) /$ Ag $(6 \mathrm{~nm}) / \mathrm{ITO}(12 \mathrm{~nm})$ to decrease the sheet resistance and support both hole injection and transparency. Also, we used the $\mathrm{Al}(100 \mathrm{~nm})$ as the cathode to support a better electron injection. $\mathrm{Ta}_{2} \mathrm{O}_{5} / \mathrm{SiO}_{2}$ as DBR layers provide high reflection and low absorption. Therefore, 12 and 11 pairs of $\mathrm{Ta}_{2} \mathrm{O}_{5} / \mathrm{SiO}_{2}$ layers with a quarter wavelength optical thickness for each layer are used as the bottom mirror and

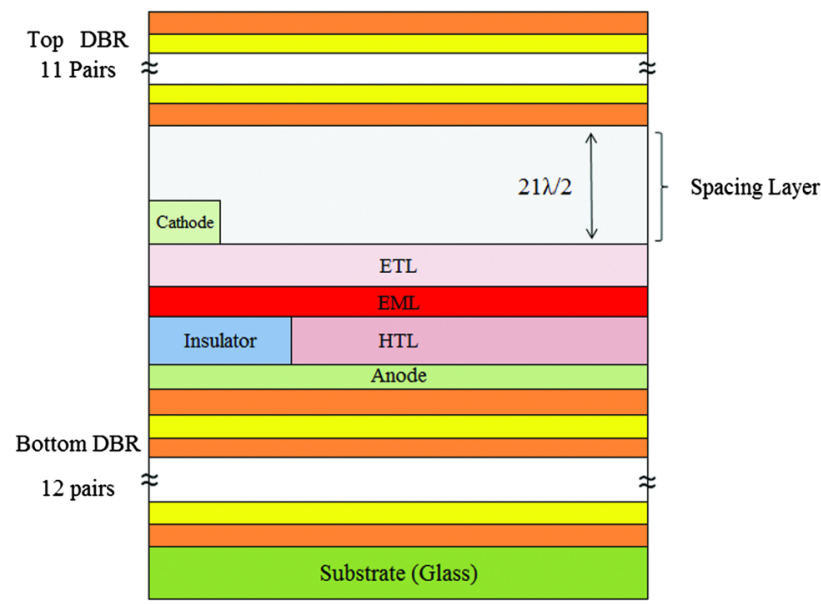

Fig. 1 Schematic structure of the proposed OVCSEL device top mirror, respectively. To improve the quality factor, a matching layer (or spacer) with a thickness of a multiple of $\lambda / 2$ between the active region and the top mirror has been used. The spacing layer can be a dielectric layer or more simply air or nitrogen if the top mirror is mounted on micromanipulators. As a result of optical modeling of the transmission spectrum, the thickness of spacing layer is derived to be $6510 \mathrm{~nm}$ corresponding to $21 \lambda / 2$ at $\lambda=620 \mathrm{~nm}$.

For the fabrication of the device structure, the bottom mirror could be made by ion beam assisted deposition (IBAD) method on the glass substrate. Then, deposition of the OLED layers onto the bottom mirror is performed. Accurate alignment of the metallic cathode is obtained using an integrated shadow mask technique and angled deposition of the aluminum layer. To prevent the destruction of the organic layers duo to the top mirror deposition, the fabricated top mirror is placed closer to the rest of the device using a micro-positioning to control its position [12].

For electrical excitation case, when an electric current is injected to the device, electrons are injected from cathode into ETL layer. The EML layer on top of the insulator is depleted of holes. The lowest unoccupied molecular orbital (LUMO) offset at the interface between EML and ETL prevents electron injection from ETL into EML. As a result, the depleted EML on top of the insulator behaves as a dielectric layer in series to the insulator, and an electron accumulation layer is formed in the ETL at the interface with EML. These electrons are transported laterally by the electric field from the cathode towards the recombination zone, i.e. the insulator edge. Hence, a micrometer-sized distance between the cathode and the light-emission zone is bridged by electrons with enhanced field-effect mobility. Near the insulator edge, electrons are injected into the EML, where they recombine with holes, injected from the anode and transported vertically through HTL. The exciton formation occurs and then radiative relaxation of these excitons to the ground state results in light-emission near the insulator edge, at a micrometer-sized distance from the metallic contact [13]. In this way, the proposed OVCSEL allows to minimize optical losses at the metal cathode.

\section{Theoretical modeling}

In this section, we investigate the dynamics of the OVCSEL optically pumped with sub-picosecond pulses. The high-quality factor and the sub-picosecond pulses pump avoid relaxation oscillation. Therefore, lasing in the OVCSEL can be modeled using the following rate equations $[10,15]$ : 
$\frac{\mathrm{d} N_{\mathrm{A}}(t)}{\mathrm{d} t}=-\frac{N_{\mathrm{A}}(t)}{\tau_{\mathrm{A}}}-\frac{N_{\mathrm{A}}(t)}{\tau_{\mathrm{F}}}$

$\frac{\mathrm{d} N_{\mathrm{D}}(t)}{\mathrm{d} t}=\frac{N_{\mathrm{A}}(t)}{\tau_{\mathrm{F}}}-\frac{N_{\mathrm{D}}(t)}{\tau_{\mathrm{D}}}-C \sigma_{\mathrm{se}} N_{\mathrm{D}}(t) N_{\mathrm{P}}(t)$

$\frac{\mathrm{d} N_{\mathrm{P}}(t)}{\mathrm{d} t}=\Gamma C \sigma_{\mathrm{se}} N_{\mathrm{D}}(t) N_{\mathrm{P}}(t)-\frac{N_{\mathrm{P}}(t)}{\tau_{\mathrm{cav}}}+\Gamma \beta \frac{N_{\mathrm{D}}(t)}{\tau_{\mathrm{D}, r}}$

where $N_{\mathrm{A}}$ and $N_{\mathrm{D}}$ denote the excited state density of $\mathrm{Alq}_{3}$ and DCM, respectively, while $N_{\mathrm{P}}$ represents the photon density of the lasing mode. Equation (1) describes the dynamics of the $\mathrm{Alq}_{3}$ molecules. The first term represents the loss mechanism due to radiative and nonradiative decay. The last term represents the loss mechanism due to Förster resonant energy transfer (FRET) to DCM molecules. Due to the delta-like pumping, the initial excited state density of $\mathrm{Alq}_{3}$ is $\eta E_{\mathrm{in}} / h v_{\mathrm{p}} v_{\mathrm{exc}}$, where $E_{\text {in }}$ is the pump energy density, $\eta$ is fraction of energy absorbed by the film, $v_{\mathrm{p}}$ is the pump frequency, $h$ is Planck constant, and $v_{\text {exc. }}$ is excitation volume. We also assume that excitation spot size is $50 \mu \mathrm{m}$ and the area of incident beam is $20 \times 10^{-6} \mathrm{~cm}^{2}$.

Equation (2) describes the dynamics of DCM molecules. The first term denotes the rate at which the DCM excitons are created due to FRET process. The second term accounts for the losses of excitons due to radiative and nonradiative decay. The third term accounts for the simulated photons; $\sigma_{\mathrm{se}}$ is the stimulated emission cross section of DCM, and $c$ is the vacuum speed of light.

Equation (3) describes the dynamics for photon in the lasing mode. The first term is proportional to number of stimulated emitted photons with the power confinement factor $\Gamma$. Power confinement factor was calculated using FDTD simulation to be 0.3 for the fundamental mode. The second term includes all cavity losses with the cavity lifetime $\left(\tau_{\text {cav }}\right)$, which is calculated from $\tau_{\text {cav }}=Q / 2 \pi v$. The last term represents the spontaneous emission photons into the laser mode with $\beta$ as fraction emitted into the laser mode.

\section{Simulation results}

The reflectance spectrum of the mirrors is shown in Fig. 2. The mirrors were designed to have a maximum reflectivity at the central emission wavelength of the active region, i.e. $\lambda_{0}=620 \mathrm{~nm}$ which is the peak emission of the $\mathrm{Alq}_{3}: \mathrm{DCM}$ guest-host system [16]. The simulated transmission spectrum of the microcavity which is calculated using transfer matrix method (TMM) is shown in Fig. 3. This figure shows that the microcavity exhibits only one transmission peak within the photoluminescence spectrum of $\mathrm{Alq}_{3}: \mathrm{DCM}$ and a quality factor up to 16,000 . Indeed, the satellite transmissions peaks are located outside of the

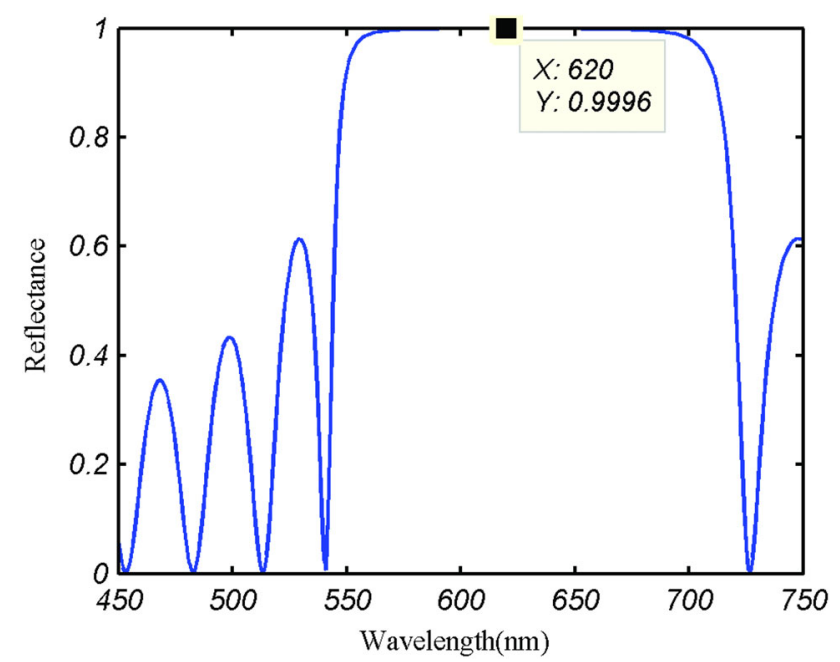

Fig. 2 Reflectance spectrum of the DBR mirror consists of 12 pairs of $\mathrm{Ta}_{2} \mathrm{O}_{5} / \mathrm{SiO}_{2}$ layers

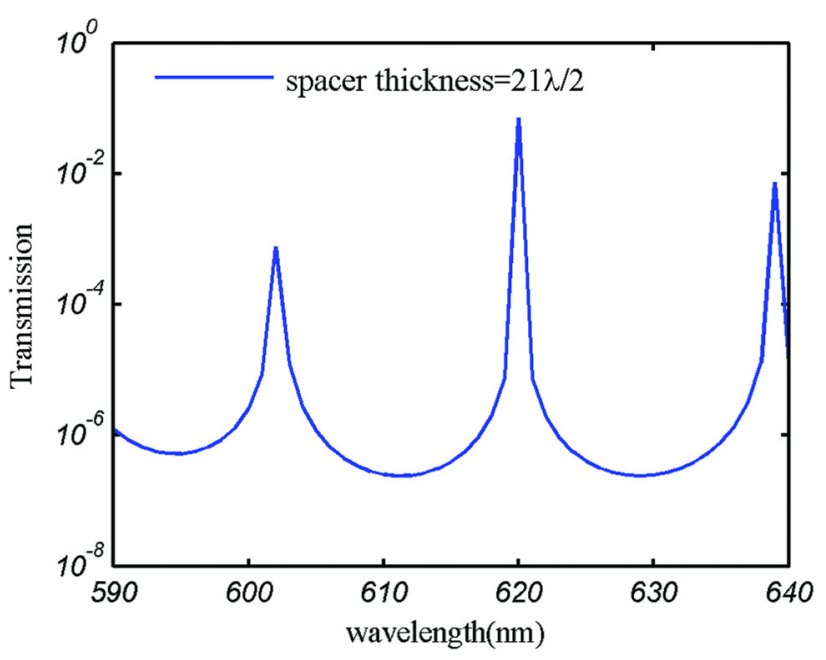

Fig. 3 Calculated transmission spectrum of the microcavity using TMM

photoluminescence spectrum of $\mathrm{Alq}_{3}: \mathrm{DCM}$. Therefore, the dynamic single-mode operations can be obtained.

For laser emission, the device is optically pumped by pulse lengths of about $200 \mathrm{fs}$, and the optical pump wavelength of $\lambda=400 \mathrm{~nm}$ is considered. When wavelength of optical pump is $400 \mathrm{~nm}$, the microcavity will have low reflection for a large range of incident angles. To evaluate the results, the coupled Eqs. (1)-(3) are numerically solved with initial condition by a fourth-order RungeKutta algorithm. The simulation parameters are given in Table 1 . The quality factor and confinement factor of the microcavity are determined using numerical simulation. In the calculation the materials are characterized by complex refractive indices. Other parameters such as radiative 
Table 1 Device parameters used in the numerical simulation $[10,11,13]$

\begin{tabular}{lll}
\hline Symbol & Parameter & Value \\
\hline$\lambda$ & Lasing wavelength & $620 \mathrm{~nm}$ \\
$\tau_{\mathrm{D}}$ & Radiative and non-radiative relaxations time for guest molecules & $1.3 \mathrm{~ns}$ \\
$\tau_{\mathrm{D}, r}$ & Radiative lifetime & $5 \mathrm{~ns}$ \\
$\tau_{\mathrm{F}}$ & Förster transfer time & $10 \mathrm{ps}$ \\
$\tau_{\mathrm{A}}$ & Radiative and non-radiative relaxations time for host molecules & $16 \mathrm{~ns}$ \\
$\beta$ & Spontaneous emission coupling factor & $1 \times 10^{-4}$ \\
$Q$ & Quality factor of microcavity & 16,000 \\
$\sigma_{\mathrm{SE}}$ & Stimulated emission cross section & $1 \times 10^{-16} \mathrm{~cm}^{2}$ \\
$\Gamma$ & Confinement factor & 0.3 \\
$\eta$ & Fraction of absorbed light & 0.9 \\
\hline
\end{tabular}

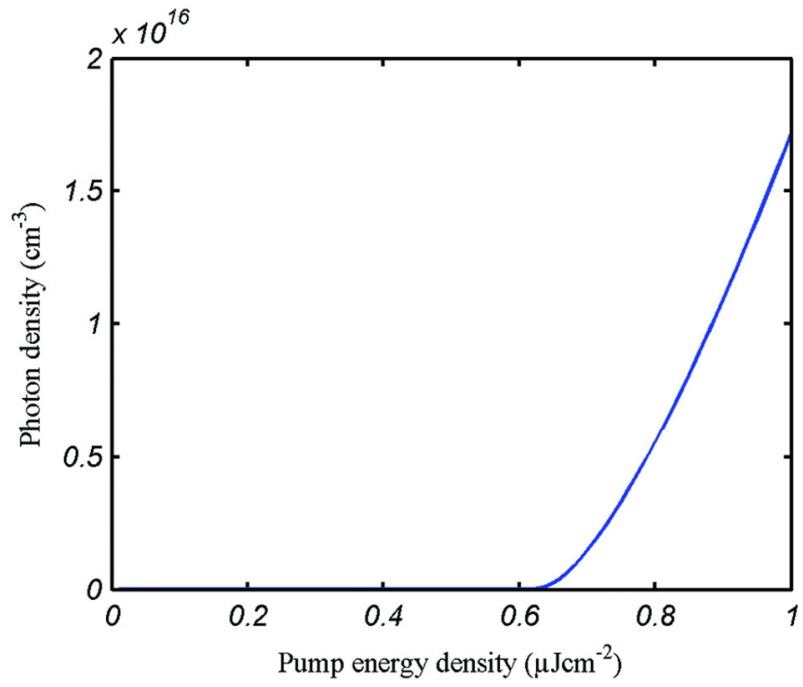

Fig. 4 Photon density peak versus pump energy densities

lifetime, Förster transfer time, and spontaneous emission coupling factor, etc., are values from the literature.

Figure 4 shows the photon density peak versus pump energy densities. This plot shows threshold at pulse energy density of about $0.64 \mu \mathrm{J} \mathrm{cm}{ }^{-2}$ (pump energy $=12.8 \mathrm{pJ}$ ). Figure 5 shows the photon density and the exciton density versus time for pump energy density of $2 \mu \mathrm{J} \mathrm{cm}{ }^{-2}$, which is above threshold. As can be seen in this figure, the excitons increase fast; however, the photon density rises to its pick when exciton density decreases abruptly.

Figure 6 shows the photon density versus time for different pump energy densities above the threshold. As one can see, both delay and output pulse width decrease with higher pump energy density, as it is well known for optical gain-switched inorganic semiconductor VCSEL and has been demonstrated already $[17,18]$.

For electrical excitation, the current density needed to achieve the same pumping which is required for lasing in the optical pumped structure can be calculated by [19]

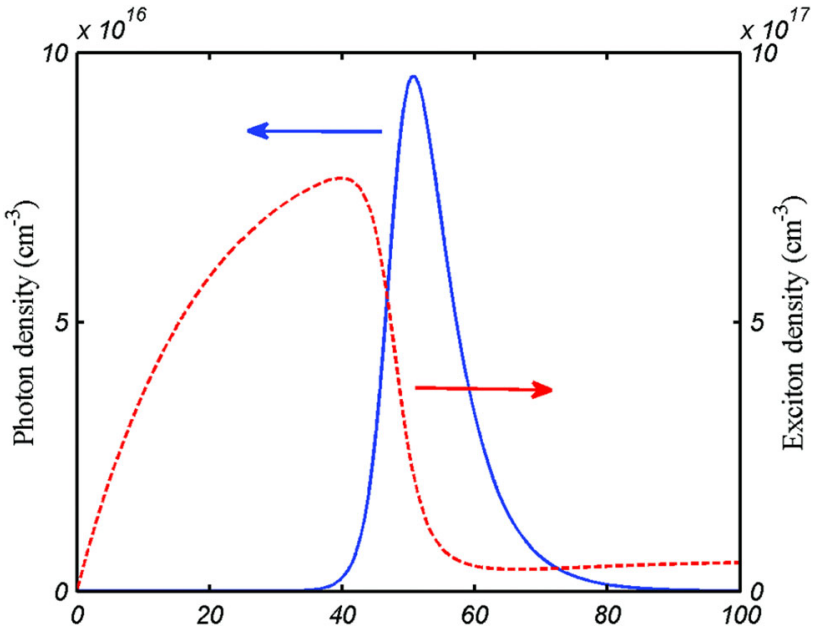

Fig. 5 Temporal evolution of the photon and exciton density for pump energy density of $2 \mu \mathrm{J} \mathrm{cm}-2$

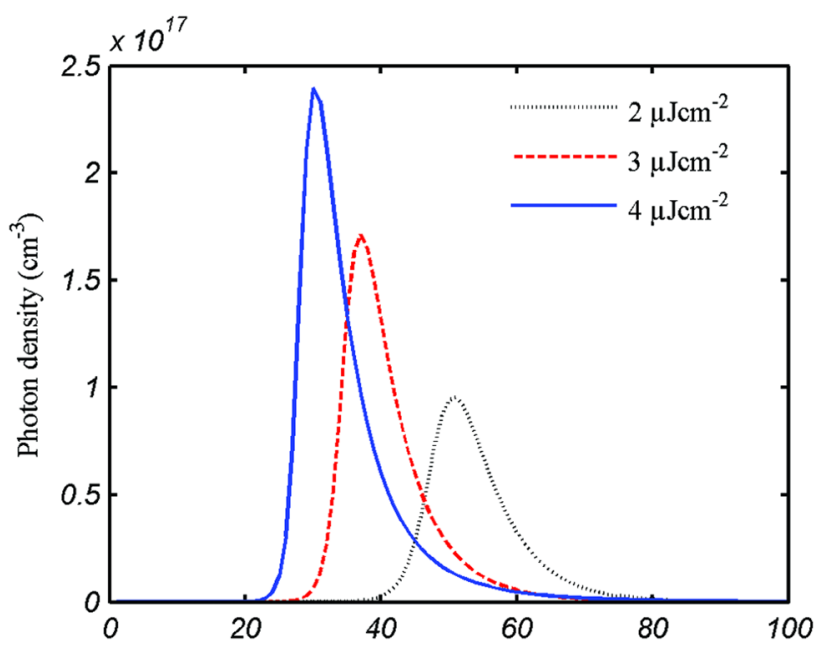

Fig. 6 Photon density versus time for different pump energy densities 
$J$ th $=\frac{2 n e}{\chi \tau_{\mathrm{D}}}$

where $n$ is number of excitons formed in the emission layer and $e$ is the electron charge. $\chi$ is the ratio of radiative singlet excitons to the total number of excitons formed by electrical injection, which is assumed to be 0.25 . Using Eq. (4), the electrical current density is $55 \mathrm{~A} \mathrm{~cm}^{-2}$ for lasing threshold.

In this work, we have focused on design and simulation of a high-quality optical microcavity toward the development of an electrical pumped organic laser. In this context, it has to be noted that the optical properties of these structures depend on the preparation technique. However, practical works are under progress to demonstrate the feasibility of such devices.

\section{Conclusion}

In this paper, we have proposed a low-threshold optically pumped organic vertical-cavity surface-emitting laser. The device has the feasibility to apply both electrical and optical pumping. Moreover, the device exhibits several characteristics that promise to respect to the realization of an electrically pumped organic laser. The reduction optical losses due to the remoteness of the metallic cathode are the most important ones. The laser microcavity structure consists of an OLED with field-effect electron transport inserted in a high-quality factor double DBR. The simulated quality factor of the microcavity which has been calculated using transfer matrix method (TMM) is as high as 16,000. Also, we have investigated the threshold behaviour and ultrafast dynamics of the OVCSEL optically pumped with sub-picosecond pulses. Results from numerical simulation show that lasing threshold is of about $12.8 \mathrm{pJ} / 0.64 \mu \mathrm{J} \mathrm{cm}{ }^{-2}$ when pumped by sub-picosecond pulses of $\lambda=400 \mathrm{~nm}$ wavelength light. This optically pumped laser threshold would correspond to a laser current density as high as $55 \mathrm{~A} \mathrm{~cm}^{-2}$. Therefore, this structure might be a promising route towards the realization of an electrically pumped organic laser.

Open Access This article is distributed under the terms of the Creative Commons Attribution 4.0 International License (http://crea tivecommons.org/licenses/by/4.0/), which permits unrestricted use, distribution, and reproduction in any medium, provided you give appropriate credit to the original author(s) and the source, provide a link to the Creative Commons license, and indicate if changes were made.

\section{References}

1. Gartner, C., Karnutsch, C., Pflumm, C., Lemmer, U.: Numerical device simulation of double-heterostructure organic laser diodes including current-induced absorption processes. IEEE J. Quantum Electron. 43, 1006-1017 (2007)

2. Tessler, N., Denton, G.J., Friend, R.H.: Lasing from conjugatedpolymer microcavities. Nature 382, 695-697 (1996)

3. Kena-Cohen, S., Forrest, S.: Room-temperature polariton lasing in an organic single-crystal microcvity. Nat. Photonics 4, 371-375 (2010)

4. Akselrod, G.M., Young, E.R., Bradley, M.S., Bulovic, V.: Lasing through a strongly-coupled mode by intra-cavity pumping. Opt. Express 21, 12122-12128 (2013)

5. Schneider, D., Rabe, T., Riedl, T., Dobbertin, T., Kroger, M., Becker, E., Johannes, H., Kowalsky, W., Weimann, T., et al.: Laser threshold reduction in an all-spiro guest-host system. Appl. Phys. Lett. 85, 1659-1661 (2004)

6. Rose, A., Zhu, Z., Madigan, C.F., Swager, T.M., Bulovic, V.: Sensitivity gains in chemo sensing by lasing action in organic polymers. Nature 434, 876-879 (2005)

7. Riechel, S., Wittwer, V., Scherf, U.: A nearly diffraction limited surface emitting conjugated polymer laser utilizing a two-dimensional photonic band structure. Appl. Phys. Lett. 77, 2310-2312 (2000)

8. Gourdon, F., Chakaroun, M., Fabre, N., Solard, J., Cambril, E., Yacomotti, A.M., Bouchoule, S., Fischer, A., Boudrioua, A.: An optically pumped lasing from organic two-dimensional planar photonic crystal microcavity. Appl. Phys. Lett. 100, 213304-213307 (2012)

9. Shapira, O., Kuriki, K., Orf, N.D., Abouraddy, A.F., Benoit, G., Viens, J.F., Rodriguez, A., Ibanescu, M., Joannopoulos, J.D., Fink, Y., et al.: Surface-emitting fiber lasers. Opt. Express 14, 3929-3935 (2006)

10. Deotare, P.B., Mahony, T.S., Bulovic, V.: Ultracompact lowthreshold organic laser. ACS Nano 8, 11080-11085 (2014)

11. Shayesteh, M.R., Darvish, G., Ahmadi, V.: A low-threshold highindex-contrast grating (HCG) - based organic VCSEL. Opt. Laser Technol. 75, 173-176 (2015)

12. Chakaroun, M., Cons, A., Fabre, N., Gourdon, F., Solard, J., Fischer, A., Boudrioua, A., Lee, C.C.: Optimal design of a microcavity organic laser device under electrical pumping. Opt. Express 19, 493-505 (2011)

13. Schols, S., Verlaak, S., Rolin, C., Cheyns, D., Genoe, J., Heremans, P.: An organic light-emitting diode with field-effect electron transport. Adv. Funct. Mater. 18, 136-144 (2008)

14. Zhong, G.Y., He, J., Zhang, S.T., Xu, Z., Xiong, Z.H., Shi, H.Z., Ding, X.M.: In situ photoluminescence investigation of doped Alq. Appl. Phys. Lett. 80, 4846-4848 (2002)

15. Koschorreck, M., Gehlhaar, R., Lyssenko, V.G., Swoboda, M., Hoffmann, M., Leo, K.: Dynamics of a high-Q vertical cavity organic laser. Appl. Phys. Lett. 87, 181108-181110 (2005)

16. Punke, M., Mozer, S., Stroisch, M., Gerken, M., Bastian, G., Lemmer, U.: Organic semiconductor devices for micro-optical application. Proc. SPIE 6185(618505), 1-13 (2006)

17. Melcer, L.G., Karin, J.R., Nagarajan, R., Bowers, J.E.: Picosecond dynamics of optical gain switching in vertical cavity emitting lasers. IEEE J. Quantum Electron. 27(6), 1417-1425 (1991)

18. Demokan, M.S., Nacaroglu, A.: An analysis of gain-switched semiconductor lasers generating pulse-code-modulated light with a high bit rate. IEEE J. Quantum Electron. 20(9), 1016-1022 (1984)

19. Kozlov, V.G., Parthasarathy, G., Burrows, P.E., Khalfin, V.B., Wang, J., Chou, S.Y., Forrest, S.R.: Structures for organic diode laser and optical properties of organic semiconductors under intense optical and electrical excitation. IEEE J. Quantum Electron. 36, 18-26 (2000) 